\title{
Improved nitrogen use efficiency in transgenic sugarcane: phenotypic assessment in a pot trial under low nitrogen conditions
}

\author{
S. J. Snyman • E. Hajari $\cdot$ M. P. Watt • \\ Y. Lu • J. C. Kridl
}

Received: 15 January 2015/Revised: 10 February 2015/ Accepted: 10 February 2015/Published online: 17 February 2015

(C) Springer-Verlag Berlin Heidelberg 2015

\begin{abstract}
Key message Sugarcane lines transformed with an alanine aminotransferase gene demonstrated an improved nitrogen use efficiency compared with untransformed controls in a pot trial under low nitrogen conditions.
\end{abstract}

\section{Introduction and aims}

Plant nitrogen $(\mathrm{N})$ nutrition has been the focus of recent efforts in crop improvement via genetic engineering. Improved $\mathrm{N}$ use efficiency (NUE) by overexpression of a barley alanine aminotransferase gene (AlaAT) in canola (Good et al. 2004) and rice (Shrawat et al. 2008) is notable as increased biomass and grain yield were recorded at low $\mathrm{N}$ supply $(0.5-4 \mathrm{mM})$ in hydroponics, growth chambers and field conditions. However, several challenges exist in assessing this 'phenotype' in transgenic plants, viz.:

Communicated by N. Stewart.

S. J. Snyman $(\varangle) \cdot$ E. Hajari

South African Sugarcane Research Institute (SASRI),

Private Bag X02, Mount Edgecombe 4300, KwaZulu-Natal,

South Africa

e-mail: sandy.snyman@ sugar.org.za

S. J. Snyman · E. Hajari · M. P. Watt

School of Life Sciences, University of KwaZulu-Natal,

Westville Campus, Private Bag X54001,

Durban 4000, KwaZulu-Natal, South Africa

Y. Lu · J. C. Kridl

Arcadia Biosciences Inc., 202 Cousteau Place, Suite 200, Davis,

CA 95618, USA incomplete synthesis of data that consider both $\mathrm{N}$ utilisation (NUtE; biomass/plant $\mathrm{N}$ content) and $\mathrm{N}$ uptake (NUpE; plant N/N supplied); lack of appropriate controls; and comparisons between pot and field evaluations (Brauer and Shelp 2010). To begin to undertake NUE studies with sugarcane-a monocotyledonous, non-cereal, vegetatively propagated crop that is only replanted every $3-10$ years-a pot trial protocol was established using five transgenic AlaAT-expressing lines of a South African sugarcane cultivar, NCo376.

\section{Methodological approach}

Transformation

Embryogenic callus of NCo376 was co-bombarded with two constructs, the first containing the barley alanine aminotransferase cDNA and rice OsAnt1 promoter (Shrawat et al. 2008), the second with the nptII selectable marker gene and maize ubiquitin promoter (Snyman 2004).

Expression analyses: RNA was extracted from 4-monthold leaf and root material using a CTAB protocol and cDNA synthesized using Maxima reverse transcriptase (Thermo Scientific, Waltham, MA). Specific primers were designed for barley AlaAT: forward 5'-CTAATGCT GATGACATTTTTCTC- $3^{\prime}$ and reverse $5^{\prime}$-CAGCCCGTC GATTCATTG- $3^{\prime}$. Sugarcane tubulin A was used as the reference gene with primers 5'-AGGCCCAACTACTC

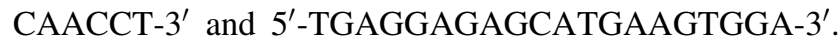
Real-time PCR was conducted in a $20 \mu 1$ reaction [50 ng cDNA, $200 \mu \mathrm{M} \mathrm{MgCl}_{2}, 200 \mathrm{nM}$ of each primer and the DyNAmo $^{\text {TM }}$ HS SYBR Green qPCR Kit (Thermo Scientific, Waltham, MA)] using an ABI 7300 real-time PCR machine (Applied Biosystems Inc., Foster City, CA). 


\section{Pot trial}

Transgenic and wild-type control plants were multiplied in vitro and planted in 3L pots containing coarse river sand in a glasshouse under ambient light and $22-26^{\circ} \mathrm{C}$. Controls comprised untransformed NCo376 and N19, regarded as low and high NUE cultivars, respectively, as per present South African industry pot trial rankings (Schumann et al. 1998). Pots were immersed in metal troughs (18 pots/ trough; random block design), which contained liquid nutrients (Schumann et al. 1998; but without N), the surface of which was covered with thick black plastic. Nitrogen (as $\mathrm{NH}_{4} \mathrm{NO}_{3}$ ) was supplied to the troughs in the liquid medium as follows: (1) month $1-1.2 \mathrm{mM} \mathrm{N}$ every week; (2) month 2-1.2 mM N every 2 weeks; (3) month 3$0.6 \mathrm{mM}$ N every 2 weeks; (4) month $4-0.3 \mathrm{mM} \mathrm{N}$ every 2 weeks. This regime and trial duration was based on an evaluation of NUE in Australian genotypes over 3 months in pots where $\mathrm{N}$ was supplied at limiting $(0.4 \mathrm{mM})$ and not-limiting (10 mM) levels (Robinson et al. 2007). Below- and above-ground dry biomass were recorded after 4 months. Total $\mathrm{N}$ analysis was undertaken by the SASRI Fertiliser Advisory Service with a LECO combustion analyser (TruSpec CN, LECO Corporation, Michigan, USA). Data were tested for normality using the ShapiroWilk test and subsequently analysed using ANOVA and Sidak post hoc tests. Means were compared across all tested lines for each parameter measured. Statistical analyses were done using the GenStat software package (version 14; VSN International, UK).

\section{Findings}

A range of AlaAT expression was detected in leaves and roots (Table 1). Although NUE 5 had the highest transcript level in both of these organs, it had the lowest (and not significantly different from the NCo376 control) calculated NUE of the transgenic lines, confirming that high expression does not always result in a desired 'phenotype' (Brauer and Shelp 2010). Lines NUE 23 and NUE 57 had high NUE, but showed high expression of the transgene in leaf and root materials, respectively, emphasising the difficulty in relating expression data to a NUE phenotype.

Although no difference was recorded for stalk diameter, both shoot height and tiller number (results not shown) differed for the seven lines tested, with NUE 23 and NUE 57 displaying the significantly greater shoot heights. These morphological characteristics contributed to the high biomass displayed by lines NUE 9, 23, 43 and 57-all significantly higher than the wild type NCo376 control (Table 1). Furthermore, the biomass produced by NUE 9, 23 and 57 transgenic lines was similar to that of the

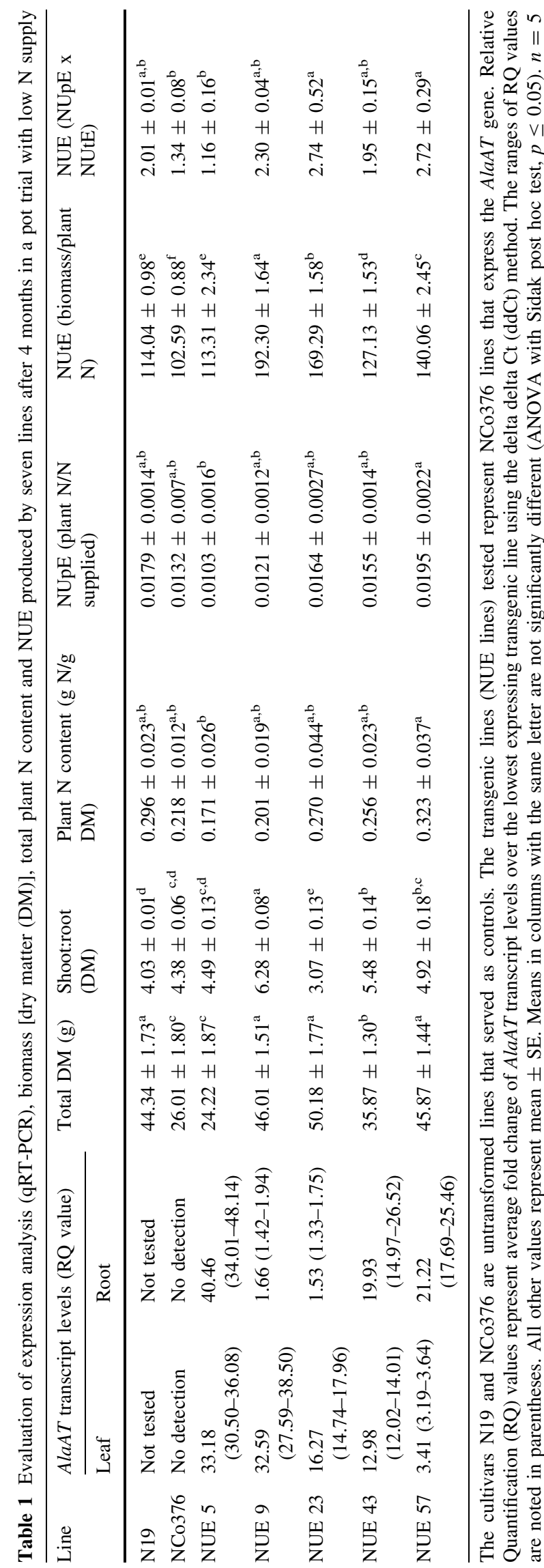


untransformed high NUE control N19. Although NUE 23 exhibited the highest shoot height and one of the highest recorded total dry mass, its shoot:root ratio was significantly lower than that of the other lines. This increased allocation of biomass to the roots was clearly evident as a large mat of roots protruding from the pots when the trial was harvested. This highlights the importance of assessing above- and below-ground biomass in sugarcane where the desired high NUE is ideally channelled towards the former.

The NUEs of lines 23 and 57 were significantly improved when compared with that of the wild type, low NUE, NCo376 control. Further, the similarity between their NUE values and that of the high NUE N19 control supports the potential of the transgenic approach. These results are promising considering the polyploid nature of the sugarcane genome and the small sample size in this study. Further, there were differences amongst the transgenic lines regarding the contribution of their $\mathrm{NUpE}$ and NUtE to NUE, and such genotypes may prove valuable as tools for the understanding of these processes.

Future work will consider NUE under field conditions and different $\mathrm{N}$ regimes, where yield parameters such as sucrose in a mature crop (12-18 months old) can be determined. However, given the constraints in controlling $\mathrm{N}$ supply in a field situation, we consider the pot trial conditions assessed in this study as adequate and useful to rapidly screen and detect differences amongst lines relatively quickly (4 months) after identifying a putative transgenic sugarcane event.

Author contribution statement EH and SJS designed the pot trial. EH conducted the pot trial. YL and JK designed and undertook the qRT-PCR analysis. SJS and
MPW wrote the focus article. All authors read and approved the article.

Acknowledgments We acknowledge and thank the South African Sugarcane Research Institute (SASRI), University of KwaZulu-Natal (UKZN) and the National Research Foundation of South Africa (Grant nos. 85573 and 85414) for funding. E. Hajari thanks the UKZN College of Agriculture, Engineering and Science for a postdoctoral scholarship. Technical assistance provided by SASRI staff $\mathrm{G}$ Meyer, N Moodley-Padaychee, N Keeping and S Mhlongo is acknowledged. The AlaAT genetic construct was provided by Arcadia Biosciences Inc. under licence to SASRI.

Conflict of interest The authors declare that they have no conflict of interest.

\section{References}

Brauer EK, Shelp BJ (2010) Nitrogen use efficiency: re-consideration of the bioengineering approach. Botany 88:103-109

Good AG, Johnson SJ, De Pauw S, Carroll RT, Savidov N, Vidmar J, Lu Z, Taylor G, Stroeher V (2004) Engineering nitrogen use efficiency with alanine aminotransferase. Can J Bot 85:252-262

Robinson N, Fletcher A, Whan A, Critchley C, von Wire N, Lakshmanan P, Schmidt S (2007) Sugarcane genotypes differ in internal nitrogen use efficiency. Funct Plant Biol 34:1122-1129

Schumann AW, Meyer JH, Nair D (1998) Evidence for different nitrogen use efficiencies of selected sugarcane varieties. Proc $S$ Afr Sug Technol Ass 72:72-80

Shrawat AK, Carroll RT, DePauw M, Taylor GJ, Good AG (2008) Genetic engineering of improved nitrogen use efficiency in rice by the tissue-specific expression of alanine aminotransferase. Plant Biotech J 6:722-732

Snyman SJ (2004) Transformation of sugarcane. In: Curtis IS (ed) Transgenic crops of the world-essential protocols. Kluwer Academic Publishers, The Netherlands, pp 103-114. ISBN: $1-4020-2332-4$ 